\title{
Pseudotumor hemofílico craneal. Caso Clínico
}

\author{
F. Conde; E. Lazo; J. Lourido y J. Domínguez
}

Servicio de Neurocirugía. Hospital Universitario Nuestra Señora de Candelaria. Santa Cruz de Tenerife.

\section{Resumen}

El pseudotumor hemofílico se define como un hematoma encapsulado que aumenta de volumen de forma progresiva por episodios de hemorragia recurrente. Es una complicación poco frecuente de la hemofilia que se presenta en el $1-2 \%$ de los pacientes con déficit moderado o grave de Factor VIII o IX. Se localiza con más frecuencia en los huesos largos de extremidades inferiores y pelvis. Presentamos el caso clínico de un varón de 21 años de edad con déficit moderado de Factor VIII (19\% de actividad de factor VIII) que desarrolló un pseudotumor en el cráneo. Hemos encontrado únicamente tres casos de pseudotumor hemofílico craneal descritos en la literatura médica.

PALABRAS CLAVE: Pseudotumor. Hemofilia. Cirugía.

\section{Cranial hemophilic pseudotumor}

\section{Summary}

The haemophilic pseudotumor is defined as an encased hematoma that increases of volume progressively by episodes of recurrent hemorrhage. It is a rare complication of haemophilia occurring in $1-2 \%$ of patients with moderate or severe factor VIII or IX deficiency. Its more frequent location is in the long bones of low extremities and pelvis. We report a case of a 21-year-old man with moderate deficiency of factor VIII (19\% of normal factor VIII activity) that developed a pseudotumor in the cranium. To our knowledge, this is the third case of the cranial hemophilic pseudotumor in medical literature.

KEY WORDS: Pseudotumor. Haemophilia. Surgery.

\section{Introducción}

La primera descripción de pseudotumor hemofílico data de 1918. Starker ${ }^{19}$. describió un pseudotumor afectando al fémur de un varón de 14 años de edad. Tras esta primera descripción se han publicado unos 100 casos de pseudotumor hemofílico extracraneal, estableciéndose criterios para su diagnóstico y tratamiento $13,3,6,10,13,16$.

El pseudotumor hemofílico es un hematoma encapsulado que aumenta de volumen de forma progresiva debido a episodios de hemorragia recurrente. Es una complicación grave de la hemofilia que se presenta en el 1-2\% de los pacientes con déficit moderado o grave de Factor VIII ó IX IX,4,20,21. $^{2}$.

La localización más frecuente está en los huesos largos de las extremidades inferiores y pelvis, mientras la órbita, mandíbula, clavícula y pequeños huesos de la mano es menos frecuente $\mathrm{e}^{3,9,10,13,16}$. Radiológicamente puede ocasionar destrucción ósea e incluso osteoformación ${ }^{6,7,22}$.

El tumor pseudohemofílico de cráneo es muy poco frecuente. Según nuestro conocimiento, éste es el tercero publicado con afectación del cráneo. El primero fue descrito en 1993 por Horton et al. ${ }^{16}$ y el segundo en 1996 por Ki Bum et al. ${ }^{14}$.

Presentamos un caso de pseudotumor hemofílico, tratado quirúrgicamente, localizado en región parietal de la bóveda craneal en un varón de 21 años de edad afecto de hemofilia A moderada.

\section{Caso Clínico}

Varón de 21 años de edad, que ingresa en el Servicio de Neurocirugía por presentar nódulos en región parietal izquierda que han aumentando de volumen en los últimos 7 meses.

Como antecedentes personales relevantes destacan: hemofilia A moderada e intervención quirúrgica de escafocefalia en la infancia (craniectomía+bandas de silicona+fijación ósea con hemoclips). Presentó hematoma intraparenquimatoso cerebral espontáneo que fue tratado de forma conservadora. No existen episodios conocidos de

Recibido: 18-02-05. Aceptado: 12-04-05 


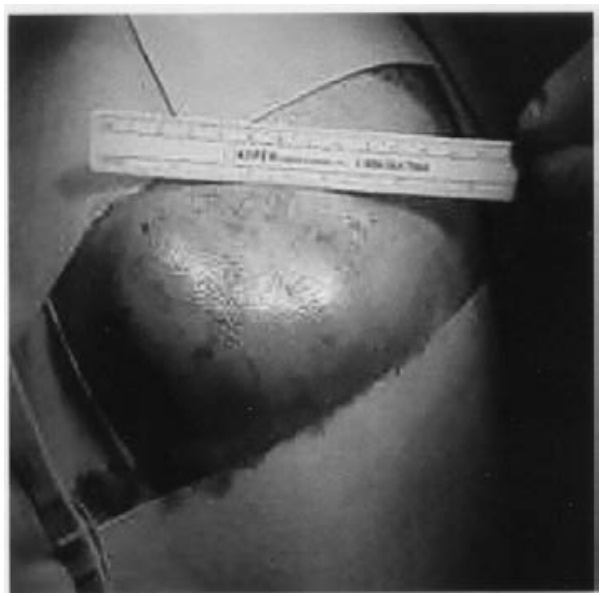

Figura 1. Tumoración indolora, no pulsátil, de superficie lisa, cubierta de scalp normal y con un tamaño de $5 \times 3 \times 3$ cm en la región parietal.

traumatismo.

El examen físico reveló una tumoración elástica indolora, no pulsátil, de superficie lisa, cubierta de "scalp" normal y con un tamaño de $5 \times 3 \times 3 \mathrm{~cm}$. en la región parietal izquierda (Fig.1). El examen neurológico fue normal.

Los hallazgos de laboratorio fueron: hemoglobina, $16 \mathrm{~g} /$ $\mathrm{dl}$; hematocrito, 50\%; leucocitos, 6710 , con un recuento diferencial normal; plaquetas, 211000; actividad de protrombina, 97\%; TTPA: 59seg. Factor VIII: 19\%.

La radiografía simple y TAC de cráneo mostraron una lesión osteolítica de márgenes escleróticos a nivel parietal izquierdo con erosión de la tabla externa e interna y expansión del díploe (Fig.2). La RMN revela un nódulo parietal en el mismo lado, bien delimitado, con distintas áreas de señal en su interior, con hemoglobina en distintos estadios de evolución: alta densidad de señal para la metahemoglobina y baja densidad de señal para la desoxihemoglobina (Fig.3).

La TAC de tórax, abdomen y pelvis, sasí como la gammagrafía ósea, descartaron tumor o metástasis ósea. El paciente recibió $50 \mathrm{U} / \mathrm{kg} / \mathrm{d}$ de Factor VIII previo a la cirugía lo cual aumentó su nivel de Factor VIII al 89\% del normal. A lo largo de la operación recibió una infusión intravenosa continua de $5 \mathrm{U} / \mathrm{kg} / \mathrm{h}$ de Factor VIII.

\section{Intervención quirúrgica}

Bajo anestesia local se realizó incisión del "scalp" sobre la tumoración y mediante disección subperióstica se observó elevación y erosión de la tabla externa, conteniendo el díploe tejido fibroso cigatricial en torno a un hematoma en distintos estadios de organización y, además, tejido plástico (bandas de silicona). La tabla interna estaba adelgazada, con abalonamiento irregular y defecto óseo.

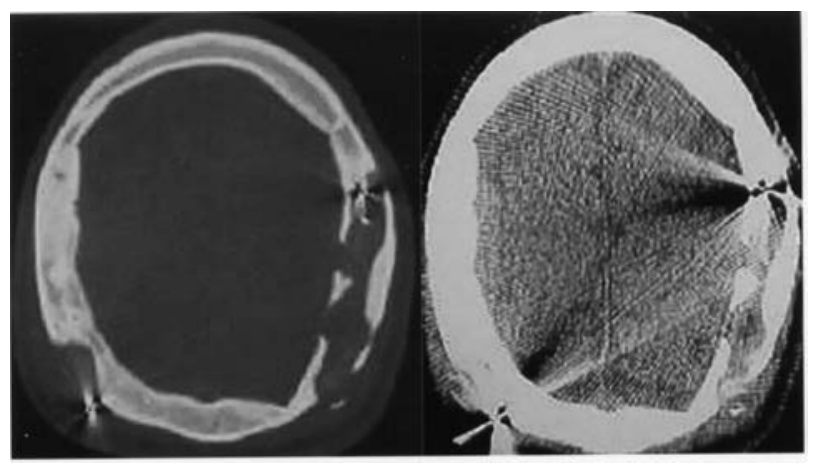

Figura 2. TAC de cráneo mostrando una lesión osteolítica en región parietal izquierda a nivel del díploe con erosión de la tabla externa e interna y expansión del diploe.

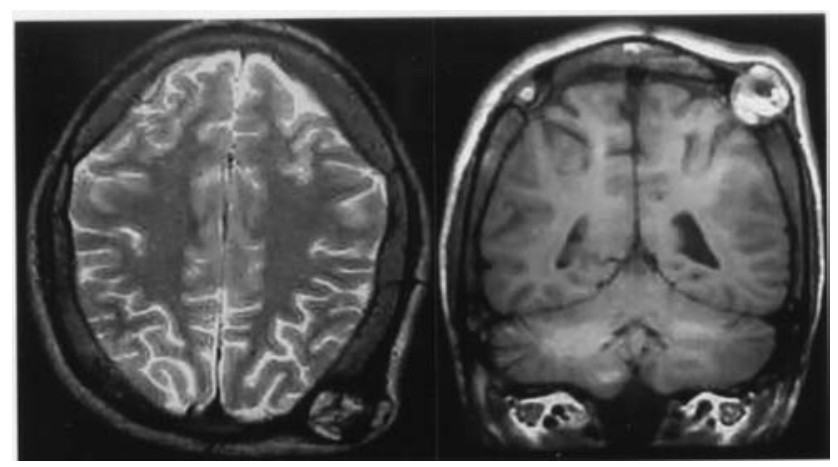

Figura 3. RMN T2 axial (A) y T1 coronal (B) revelando nódulo parietal izquierdo, extraaxial, con distintas áreas de señal en su interior representando la hemoglobina en diferentes estadios de evolución: alta densidad de señal para la metahemoglobina y baja densidad de señal para la desoxihemoglobina.

Se extirpó el hematoma, el tejido granular y cuerpo extraño (silicona) así como la tabla externa adelgazada y elevada, hasta llegar a hueso de grosor y aspecto normal mediante curetaje óseo.

El examen histológico confirmó la presencia de hematoma antiguo y tejido de granulación.

Tras la cirugía, el Factor VIII se mantuvo en el $80 \%$ de la actividad normal hasta que se retiró el material de sutura.

\section{Discusión}

Tres tipos de pseudotumor se han descrito en pacientes con hemofilia.

El primero ocurre en los músculos, debido a una fístula arteriovenosa. La masa aumenta lentamente, desarrolla una cápsula fibrosa y destruye los tejidos circundantes, incluyendo el hueso, mediante necrosis por presión o disección de su inserción ósea. Suele ocurrir en iliopsoas, 
cuadriceps, tríceps y glúteo mayor ${ }^{1,7,11,13}$. El segundo tipo se origina por hemorragia subperióstica erosionando huesos tales como el ilium, fémur y tibia ${ }^{1,3,6,7,11,13}$. El tercer tipo, y menos frecuente, se origina dentro de huesos tales como el calcáneo, el ilium y el cráneo. Mediante repetidas o continuadas hemorragias, el pseudotumor aumenta de tamaño, causando expansión y adelgazamiento de la cortical ósea, que lo predispone a posterior fractura y hemorragia ${ }^{7,8,11,13,14}$. El pseudotumor en cráneo presenta riesgo de rotura y hemorragia al espacio subgaleal o epidural, necrosis de "scalp", septicemia secundaria y compresión directa sobre el parénquima cerebral ${ }^{14}$.

La gravedad de la hemofilia es constante para un mismo paciente, pero varía de un enfermo a otro. En la forma leve, el paciente tiene un nivel funcional en plasma de Factor VIII o IX del $20-60 \%$. En la forma moderada, el nivel en plasma es de 5-20\% y el sangrado puede ocurrir durante la cirugía o después de un traumatismo. En la hemofilia grave los niveles en plasma de Factor VIII o IX son inferiores al $5 \%$ pudiendo sufrir episodios de hemorragia espontánea o después de un traumatismo leve ${ }^{1}$.

El pseudotumor puede asemejarse, radiológicamente, a otras lesiones tales como la osteomielitis, sarcoma de Ewing, osteosarcoma, metástasis, absceso tuberculoso, quiste óseo aneurismático y plasmocitoma. También puede simular un hematoma epidural o subdural calcificado ${ }^{3,13,15}$.

Para su diagnóstico, la radiografía simple, la TAC y los ultrasonidos son útiles pero la Resonancia magnética es el método de elección ${ }^{10,12,15,16,21}$ por su mayor sensiblidad para detectar zonas de hematoma en distintos estadios de evolución y ofrecer imágenes multiplanares ${ }^{12}$.

El tratamiento consiste en administración de Factor VIII o IX e inmovilización. La cirugía se reserva para grandes lesiones que no responden al tratamiento conservador, producen necrosis de la piel, compresión neurovascular, presentan un crecimiento progresivo o para confirmar el diagnóstico ${ }^{4,6,12,15,21}$ tal como sucedió en nuestro caso, ya que los antecedentes de cirugía previa hacían difícil la interpretación de las imágenes radiológicas. La embolización arterial se ha descrito en el tratamiento de un gran pseudotumor en el hueso iliaco ${ }^{20}$. También se ha usado el tratamiento con radioterapia que, al parecer, limita la expansión de la lesión aunque su mecanismo de acción permanece en controversia ${ }^{3,4,11,13,16}$. Su uso se ha reservado para aquellos casos en los que existe en sangre el inhibidor del Factor VIII que es responsable de hemorragias difíciles de controlar incluso tras administrar tratamiento sustitutivo con Factor VIII.

\section{Bibliografía}

1. Abell, J.M., Bailey, R.W.: Hemophilic pseudotumor: Two cases occurring in siblings. Arch Surg 1960; 81: 569581.

2. Arnold, W.D., Hilgartner, M.W.: Hemophilic arthropathy: Current concepts of Pathogenesis and management. J Bone Joint Surg Am 1977; 59: 287-305.

3. Brant, E.E., Jordan, H.H.: Radiologic aspects of hemophilic pseudotumors in bone. Am J Roentgenol 1972; 115: 525-539.

4. Castañeda, V.L., Parmley, R.T., Bozzini, M., Feldmeier, J.J.: Radiotherapy of pseudotumors of bone in hemophiliacs with circulating inhibitors to factor VIII. Am J Hematol 1991; 36: 55-59.

5. Chorobski, J., Davis, L.: Cyst formations of the skull. Surg Gynecol Obstet 1934; 58: 12-31.

6. Coon, W.W., Penner, J.A.: Management of abdominal hemophilic pseudotumor. Surgery 1981; 90: 735-740.

7. Fernández de Valderrama, J.A., Matthews, J.M.: The haemophilic pseudotumour or haemophilic subperiosteal haematoma. J Bone Joint Surg Br 1965; 47: 256-265.

8. Ghormley, R.K., Clegg, R.S.: Bone and joint changes in hemophilia: With report of cases of so-called hemophilic pseudotumor. J Bone Joint Surg Am 1948; 30: 589-600.

9. Gilbert, M.S.: Characterizing the hemophilic pseudotumor. Ann N Y Acad Sci 1975; 240: 311-315.

10. Guilford, W.B., Mintz, P.D., Blatt, P.M., Staab, E.V.: CT of hemophilic pseudotumors of the pelvis. Am Roentgenol 1980; 135: 167-169.

11. Hilgartner, M.W., Arnold, W.D.: Hemophilic pseudotumor treated with replacement therapy and radiation. J Bone Joint Surg Am 1975; 57: 1145-1146.

12. Horton, D.D., Pollay, M., Wilson, D.A., Brandon, F.M., Sexauer, C.L.: Cranial hemophilic pseudotumor: Case report.J. Neurosurg 1993; 79: 936-938.

13. Jensen, P.S., Putman, C.E.: Hemophilic pseudotumor: Diagnosis, treatment and complications. Am J Dis Child 1975; 129: 717-719.

14. Kí Bum Sim, M.K., Seung kuan Hong, M.D.: Cranial hemophilic pseudotumor: Case report. Neurosurgery 1996; 39: 1239-1242.

15. Liu, S.S., White, W.L., Johnson, P.C., Gauntt, C.: Hemophilic pseudotumor of the spinal canal: Case report. J Neurosurg 1988; 69: 624-627.

16. Meyers, L., Hakami, N.: Pseudotumor of hemophilia in the orbit: The role of radiotherapy in management. Am J Hematol 1986; 19: 294-296.

17. Palatinsky, E., Liccardo, G., Di Lorenzo, N.: Chronic subperiosteal hematoma of the skull in an adult: A case report. Neurosurgery 1986; 19: 294-296.

18. Sato, K., Kubota, T., Kawano, H.: Chronic diploic hematoma of the parietal bone: Case report. J Neurosurg 1994; 
80: 1112-1115.

19. Starker, L.: Knochenusur durch ein haemophiles subperiostales haematoma. Mittl Grenzgeb Med Chir 1918; 31: $381-415$

20. Wessler, S., Avioli, L.V.: Changes in surgical management of hemophiliacs: Pseudotumor of the ilium. JAMA 1968; 206: 2292-2296.

21. Wilson, D.A., Prince, J.R.: MR imaging of hemophilic pseudotumors. Am J Roentgenol 1988; 150: 349-350.

22. Yuasa, H., Watanabe, H., Uemura, Y., Tokito, S.:
Intraosseous hematoma of the skull: Case report. Neurosurgery 1992; 30: 776-778.

Conde, F.; Lazo, E.; Lourido, J.; Domínguez, J.: Pseudo-tumor hemofílico craneal. Neurocirugía 2006; 17: 158-161.

Dirección postal: Francisco Javier Conde Garcia. Servicio de Neurocirugía. Hospital Universitario Nuestra Señora de Candelaria. Carretera del Rosario, s/n. 38010 Santa Cruz de Tenerife. 